Ann. Scient. Éc. Norm. Sup.,

4e série, t. 40, 2007, p. 191 à 207.

\title{
LE BAS DU SPECTRE D'UNE VARIÉTÉ HYPERBOLIQUE EST UN POINT SELLE
}

\author{
PAR OLIVIER MOHSEN
}

RÉSUMÉ. - Besson, Courtois et Gallot ont démontré que, sur une variété compacte, une métrique hyperbolique est d'entropie minimale parmi les métriques de même volume. On montre ici qu'une métrique hyperbolique est un point selle pour le bas du spectre du revêtement universel. Plus précisément, son bas du spectre est maximal parmi les métriques conformes de même volume, et minimal dans la tranche d'Ebin.

(c) 2007 Elsevier Masson SAS

Abstract. - Besson, Courtois and Gallot proved that on a compact manifold, a hyperbolic metric has minimal entropy among the metrics of same volume. Here we show that a hyperbolic metric is a saddle point for the bottom of spectrum of the universal cover. More precisely, the bottom of its spectrum is maximal among the conformal metrics of same volume, and minimal in its Ebin class.

(C) 2007 Elsevier Masson SAS

\section{Introduction}

Soit $X$ une variété compacte qui admet une métrique localement symétrique à courbure strictement négative $g_{0}$. Dans [2] et [3], on étudie le comportement d'un invariant riemannien appelé «entropie volumique » (noté $h$ ) lorsqu'on fait varier la métrique de $X$ à volume constant. On montre alors que l'entropie volumique est minimale en $g_{0}$.

Dans ce texte, on s'intéresse à un autre invariant riemannien qui est le bas du spectre du revêtement universel de $X$ (noté $\lambda_{1}$ ). L'inégalité suivante est bien connue (voir [16]) :

$$
\lambda_{1} \leqslant \frac{h^{2}}{4}
$$

avec égalité si la métrique est localement symétrique. Il est connu que $\lambda_{1}$ admet un maximum en $g_{0}$ en restriction à la classe conforme de $g_{0}$ (à volume constant) (voir [4]). En particulier, en dimension 2 , comme toute métrique est conforme à une métrique hyperbolique, on en déduit que le $\lambda_{1}$ est un maximum en $g_{0}$. On montre ici que ce résultat n'est pas vrai en dimension $\geqslant 3$ : plus précisément, on considère deux sous-espaces de métriques, qui sont la classe conforme de $g_{0}$ et la tranche d'Ebin de $g_{0}$. Ces deux sous-espaces se coupent transversalement en $g_{0}$ et leurs espaces tangents en $g_{0}$ engendrent un supplémentaire de l'espace tangent à l'orbite de $g_{0}$ par l'action des difféomorphismes de $X$. On montre que

THÉORÈME 1. - Soit $X$ une variété compacte de dimension $\geqslant 3$ qui admet une métrique $g_{0}$ à courbure constante strictement négative. Alors le bas du spectre $\lambda_{1}$ admet un point selle en $g_{0}$ : $\lambda_{1}\left(g_{0}\right)$ est un maximum strict dans la classe conforme de $g_{0}$ à volume constant, et $\lambda_{1}\left(g_{0}\right)$ est un minimum strict local dans la tranche d'Ebin de $g_{0}$. 
La preuve repose sur une description du $\lambda_{1}$ qui fait intervenir les familles équivariantes de mesures sur le bord du revêtement universel. Cette description est donnée par le théorème 3 . Elle permet d'étudier les variations du $\lambda_{1}$ lorsqu'on fait varier la métrique autour d'une métrique hyperbolique.

Enfin, le théorème 3 permet aussi de mettre en évidence deux inégalités déjà connues, l'une étant l'inégalité (1), et l'autre reliant le bas du spectre à l'entropie stochastique, et on montre que dans les cas d'égalité, la métrique est localement symétrique (voir aussi $[16,14,10]$ ).

\section{Le bas du spectre et les familles de mesures équivariantes}

Soit $X$ une variété compacte. On suppose qu'elle peut être munie d'une métrique à courbure strictement négative. Soit $\widetilde{X} \rightarrow X$ un revêtement universel de $X$ et soit $\Gamma$ le groupe fondamental de $X$, vu comme le groupe de difféomorphisme de $\widetilde{X}$ tel que l'on puisse écrire

$$
X \simeq \Gamma \backslash \widetilde{X} .
$$

Une métrique $g$ sur $X$ se relève en une métrique sur $\widetilde{X}$ pour laquelle $\Gamma$ est constitué d'isométries.

Dans cette section, on fixe une métrique $g$ sur $X$ à courbure strictement négative. Si $f$ est une fonction $C^{\infty}$ non nulle de carré intégrable sur $\widetilde{X}$, on définit le quotient de Rayleigh de $f$ par

$$
R(f)=\int_{\widetilde{X}}|d f(x)|^{2} d x / \int_{\widetilde{X}} f^{2}(x) d x
$$

(où $|\cdot|$ et $d x$ désignent la norme et la mesure induite par la métrique).

On définit le bas du spectre de $\widetilde{X}$ pour la métrique $g$ comme la borne inférieure des quotients de Rayleigh :

$$
\lambda_{1}=\lambda_{1}(g)=\inf \{R(f) \mid f \text { non nulle de carré intégrable }\} .
$$

L'inconvenient de cette définition est qu'il n'existe pas de fonction qui minimise le quotient de Rayleigh. Mais dans notre contexte, $\widetilde{X}$ admet un quotient compact pour l'action de $\Gamma$, ce qui permet de donner une description plus précise de ce qu'est le $\lambda_{1}$.

Nous renvoyons par exemple à [6] et à [9] pour la définition du bord du groupe $\Gamma$, de la structure d'espace topologique compact de $\Gamma \cup \partial \Gamma$, de la définition du bord à l'infini $\partial \widetilde{X}$ et de sa structure höldérienne, indépendante de la métrique à courbure strictement négative sur $X$, et de l'homéomorphisme $\partial \Gamma \rightarrow \partial \widetilde{X}$ par lequel on identifie ces deux objets.

DÉFINITION 2. - On appelle famille équivariante de mesures sur $\partial \Gamma$ une famille $\mu=\left(\mu_{x}\right)$ qui à tout $x \in \widetilde{X}$ associe une mesure finie $\mu_{x}$ sur $\partial \Gamma$ telle que pour tout $x \in \widetilde{X}$ et pour tout $\gamma \in \Gamma$, on ait la relation d'équivariance

$$
\mu_{\gamma(x)}=\gamma_{*} \mu_{x}
$$

et telle que pour tout $x, y \in \widetilde{X}$, les mesures $\mu_{x}$ et $\mu_{y}$ soient équivalentes et que la fonction densité

$$
\frac{d \mu_{x}}{d \mu_{y}}(\theta)
$$

soit $C^{\infty}$ en les variables $x, y$, et que ses dérivées successives soient des fonctions höldériennes en $\theta, x, y$.

$4^{\mathrm{e}}$ SÉRIE - TOME $40-2007-\mathrm{N}^{\circ} 2$ 
Remarque. - L'hypothèse de régularité holdërienne joue un rôle caché dans cet article. Elle n'intervient en fait que lorsqu'on utilise les travaux de Ledrappier dans la preuve de notre théorème 3.

Chaque famille équivariante de mesures induit une classe d'équivalence de mesures sur $\partial \Gamma$. On dira que deux familles équivariantes de mesures sont équivalentes si elles induisent la même classe.

On définit une fonctionnelle sur l'ensemble des familles équivariantes de mesures sur $\partial \Gamma$ de la façon suivante : soit $\mu$ un tel objet, soit $o \in \widetilde{X}$ un point quelconque, soit $X_{0} \subset \widetilde{X}$ un domaine fondamental de $\Gamma$ et soit $r_{\theta}(x)$ la fonction définie par

$$
r_{\theta}(x)^{2}=\frac{d \mu_{x}}{d \mu_{o}}(\theta)
$$

Alors on définit

$$
\mathcal{R}(\mu)=\int_{X_{0}} \int_{\partial \Gamma}\left|d r_{\theta}(x)\right|^{2} d \mu_{o}(\theta) d x / \int_{X_{0}} \mu_{x}(\partial \Gamma) d x .
$$

Ce nombre ne dépend pas des choix de $o$ et de $X_{0}$. Pour s'en convaincre, il suffit de constater que la quantité

$$
\int_{\partial \Gamma}\left|d r_{\theta}(x)\right|^{2} d \mu_{o}(\theta)
$$

ne dépend pas de $o$, et qu'elle est invariante lorsque $\Gamma$ agit sur la variable $x$. Le nombre $\mathcal{R}(\mu)$ sera noté aussi parfois $\mathcal{R}_{g}(\mu)$ pour préciser la métrique sur $X$ que l'on considère. On appelle ce nombre le quotient de Rayleigh de $\mu$, à cause du théorème suivant :

THÉORÈME 3. - Soit $\nu$ une famille équivariante de mesures sur $\partial \Gamma$. Alors

$$
\lambda_{1}=\inf \{\mathcal{R}(\mu) \mid \mu \text { famille équivariante de mesures sur } \partial \Gamma \text { équivalente à } \nu\} .
$$

Afin de prouver ce théorème, on donne la définition suivante : on appelle famille équivariante de mesures sur $\Gamma$ une famille $m=\left(m_{x}\right)$ qui à tout $x \in \widetilde{X}$ associe une mesure finie $m_{x}$ sur le groupe discret $\Gamma$ telle que pour tout $x \in \widetilde{X}$ et pour tout $\gamma \in \Gamma$, on ait la relation d'équivariance

$$
m_{\gamma(x)}=\gamma_{*} m_{x}
$$

et aussi telle que pour tout $\gamma$, la fonction $r_{\gamma}(x)$ définie sur $\widetilde{X}$ par

$$
r_{\gamma}(x)^{2}=m_{x}(\{\gamma\})
$$

soit $C^{\infty}$ et de carré intégrable. Etant donné un tel objet $m$, on définit

$$
\mathcal{R}(m)=\int_{X_{0}} \sum_{\gamma}\left|d r_{\gamma}(x)\right|^{2} d x \int_{X_{0}} m_{x}(\Gamma) d x,
$$

où $X_{0}$ est un domaine fondamental pour $\Gamma$. Comme $\tilde{X}$ est la réunion disjointe des $\gamma X_{0}$ pour $\gamma \in \Gamma$, on constate alors que pour tout $\gamma_{0} \in \Gamma, \mathcal{R}(m)$ est égal au quotient de Rayleigh de la 
fonction $x \rightarrow r_{\gamma_{0}}(x)$ définie sur $\widetilde{X}$. Par conséquent

$$
\mathcal{R}(m) \geqslant \lambda_{1}
$$

Preuve du théorème 3. - lère partie : On se donne $\mu$ une famille équivariante de mesures sur $\partial \Gamma$ et on veut montrer que

$$
\mathcal{R}(\mu) \geqslant \lambda_{1} \text {. }
$$

L'idée consiste à voir $\mu$ comme une «limite faible» de familles équivariantes de mesures sur $\Gamma$ suffisamment régulières pour que l'on puisse appliquer (2).

Pour cela, nous allons d'abord faire appel à une construction due à Ledrappier : soit $C_{\theta}(x, y)$ la fonction définie par

$$
\frac{d \mu_{x}}{d \mu_{y}}(\theta)=e^{-C_{\theta}(x, y)}
$$

On a par définition la relation de cocycle

$$
C_{\theta}(x, y)+C_{\theta}(y, z)=C_{\theta}(x, z) .
$$

Dans [12], on montre que la famille de mesure $\mu$ est déterminée à une constante multiplicative près par la fonction $C_{\theta}(x, y)$, et qu'elle peut être reconstruite à partir de cette fonction. Nous rappelons ici les grandes lignes de cette construction (voir [12] pour les détails) : Pour $x, z \in \widetilde{X}$, on pose

$$
\Delta(x, z)=C_{\theta}(x, z),
$$

où $\theta$ est le point de $\partial \Gamma$ associé au rayon géodésique issu de $x$ et dirigé vers $z$. Ce rayon géodésique est unique si $x \neq z$, et sinon on pose $\Delta(x, z)=0$. Cette fonction $\Delta$ est du même ordre de grandeur que la fonction distance car la différentielle $d C_{\theta}(x, z)$ pour la variable $x$ est uniformément bornée car elle ne dépend pas de $z$ à cause de la relation de cocycle et elle est $\Gamma$-invariante. D'où il existe une constante $A$ telle que $\left|d C_{\theta}(x, z)\right| \leqslant A$ et donc $|\Delta(x, z)| \leqslant$ $A d(x, z)$. Pour $s \in \mathbb{R}$, on pose

$$
\Delta^{s}(x, z)=\Delta(x, z)+s d(x, z),
$$

et on définit les mesures $\mu_{x}^{s}$ sur $\Gamma$ par

$$
\mu_{x}^{s}=\sum_{\gamma \in \Gamma} e^{-\Delta^{s}(x, \gamma(o))} \delta_{\gamma},
$$

où $o \in \widetilde{X}$ est une origine fixée quelconque. La fonction qui, à un réel $s$, associe la mesure totale $\mu_{x}^{s}(\Gamma)$ est clairement décroissante, et il est facile de voir qu'elle est finie pour $s$ suffisamment grand et infinie pour $s$ suffisamment petit. On a en fait que $\mu_{x}^{s}(\Gamma)=+\infty$ pour $s<0$ et $\mu_{x}^{s}(\Gamma)<+\infty$ pour $s>0$ (voir [12], lemme 2, p. 112). Pour simplifier, nous supposerons de plus que ces mesures sont infinies pour $s=0$, même si cette condition n'est pas indispensable (voir [12], p. 112). Pour $z \in \widetilde{X}$, on pose $C_{z}(x, y)=\Delta(x, z)-\Delta(y, z)$. Si on fixe $x, y \in \widetilde{X}$, on a que la fonction $z \in \widetilde{X} \rightarrow C_{z}(x, y)$ se prolonge par continuité sur le bord à l'infini par $\theta \in \partial \Gamma \rightarrow C_{\theta}(x, y)$ (voir [12], p. 113), de même que la fonction de Busemann $\theta \rightarrow B_{\theta}(x, y)$ est le prolongement par continuité sur le bord de la fonction $z \rightarrow B_{z}(x, y)$, où on a posé

$4^{\text {e }}$ SÉRIE - TOME $40-2007-\mathrm{N}^{\circ} 2$ 
$B_{z}(x, y)=d(x, z)-d(y, z)$. Pour $s>0$, comme $\mu_{o}^{s}$ est une mesure finie, on peut poser

$$
\mu_{x}^{\prime s}=\frac{1}{\mu_{o}^{s}(\Gamma)} \mu_{x}^{s}
$$

On a alors les fonctions densité

$$
\frac{d \mu_{x}^{\prime s}}{d \mu_{y}^{\prime s}}(\gamma)=e^{-\left(\Delta^{s}(x, \gamma(o))-\Delta^{s}(y, \gamma(o))\right.}=e^{-\left(C_{\gamma(o)}(x, y)+s B_{\gamma(o)}(x, y)\right)}
$$

Comme les mesures $\mu_{o}^{\prime s}$ sont de masse 1 , il existe une suite de réels positifs $\left(s_{n}\right)$ tendant vers zéro telle que la suite de mesures $\mu_{o}^{\prime s_{n}}$ converge faiblement dans l'espace des mesures sur $\Gamma \cup \partial \Gamma$ vers une mesure $\mu_{o}^{\prime}$ de masse 1 . Le support de cette mesure limite est contenu dans $\partial \Gamma$, car comme on a supposé que $\mu_{o}^{0}(\Gamma)=+\infty$, alors $\mu_{o}^{s_{n}}(\Gamma)$ tend vers $+\infty$, et donc

$$
\mu_{o}^{\prime}(\{\gamma\})=\lim _{n \rightarrow \infty} \mu_{o}^{\prime s_{n}}(\{\gamma\})=\lim _{n \rightarrow \infty} \frac{e^{-\Delta^{s_{n}}(x, \gamma(o))}}{\mu_{o}^{s_{n}}(\Gamma)}=0 .
$$

Ainsi, pour tout $x$, les mesures $\mu_{x}^{\prime s_{n}}$ convergent faiblement vers une mesure $\mu_{x}^{\prime}$ sur $\partial \Gamma$ et la fonction densité $\frac{d \mu_{x}^{\prime}}{d \mu_{y}^{\prime}}$ est la restriction au bord de la limite uniforme des fonctions densités $\frac{d \mu_{x}^{\prime s_{n}}}{d \mu_{y}^{\prime s n}}$, i.e.

$$
\frac{d \mu_{x}^{\prime}}{d \mu_{y}^{\prime}}(\theta)=e^{-C_{\theta}(x, y)}=\frac{d \mu_{x}}{d \mu_{y}}(\theta)
$$

Donc $\mu^{\prime}$ est une famille équivariante de mesures proportionnelle à la famille $\mu$ de laquelle on est parti, et ainsi s'achève la construction dans [12].

On vient de voir que la famille équivariante de mesures $\mu$ est, à une constante près, la « limite faible» quand $s \rightarrow 0^{+}$des $\mu^{\prime s}$, qui sont des familles équivariantes de mesures sur $\Gamma$, mais le problème est qu'on ne peut pas calculer les quotients $\mathcal{R}\left(\mu^{\prime s}\right)$, par manque de régularité. Nous allons maintenant trouver une suite de familles équivariantes de mesures sur $\Gamma$ qui converge faiblement vers $\mu$ et dont on peut calculer les quotients de Rayleigh.

On se donne une partition de l'unité $\Gamma$-équivariante sur $\widetilde{X}$, c'est-à-dire une fonction $\rho$ positive $C^{\infty}$ à support compact sur $\widetilde{X}$ telle que $\sum_{\gamma} \rho \circ \gamma=1$. On peut alors définir $\nu$, famille équivariante de mesures sur $\partial \Gamma$, par

$$
\nu_{x}=\sum_{\gamma} \rho\left(\gamma^{-1} x\right) \gamma_{*} \mu_{o}
$$

Comme $\mu_{o}$ est la limite faible des $\mu_{o}^{\prime s}$ quand $s$ tend vers $0^{+}$, alors pour tout $x$, la mesure $\nu_{x}$ est la limite faible de

$$
\nu_{x}^{s}=\sum_{\gamma} \rho\left(\gamma^{-1} x\right) \gamma_{*} \mu_{o}^{\prime s}
$$

La famille équivariante de mesures $\nu$ est équivalente à $\mu$, donc on peut écrire

$$
\frac{d \mu_{x}}{d \nu_{x}}(\theta)=f(x, \theta) .
$$

La fontion $f$ définie sur $\widetilde{X} \times \partial \Gamma$ par cette égalité est une fonction strictement positive, $\Gamma$-invariante, et de classe $C^{\infty}$ pour la variable $x$, les dérivées successives étant continues pour 
$(x, \theta) \in \widetilde{X} \times \partial \Gamma$. On peut alors prolonger cette fonction $f$ sur $\widetilde{X} \times(\Gamma \cup \partial \Gamma)$. Une façon de construire ce prolongement (pas du tout canonique) est d'utiliser $p=\left(p_{x}\right)$, la famille équivariante de mesures de Patterson (voir [18]). Cette famille est caractérisée par le fait qu'il existe un nombre $h>0$ tel que pour tout $x$, la densité de $p_{x}$ par rapport à $p_{o}$ s'écrive

$$
\frac{d p_{x}}{d p_{o}}(\theta)=e^{-h B(x, \theta)}
$$

où $B(x, \theta)$ désigne la fonction de Busemann normalisée par $B(o, \theta)=0$. On suppose que $p$ est normalisé de sorte que $p_{o}(\partial \Gamma)=1$. On constate le fait suivant : pour toute suite $\gamma_{n} \in \Gamma$ qui converge vers un point $\theta_{0} \in \partial \Gamma$, les mesures $p_{\gamma_{n} o}$ convergent faiblement vers la mesure de Dirac en $\theta_{0}$. En effet, toutes ces mesures sont de masse 1 , et les fonctions densité $d p_{\gamma_{n} o} / d p_{o}$ convergent vers 0 uniformément sur les complémentaires des voisinages de $\theta_{0}$. Ainsi, pour tout $(x, \gamma) \in \widetilde{X} \times \Gamma$, on peut poser

$$
f(x, \gamma)=\int_{\partial \Gamma} f(x, \theta) d\left(\gamma_{*} p_{o}\right)(\theta),
$$

et ceci définit bien un prolongement continu de la fonction $f$ sur $\widetilde{X} \times(\Gamma \cup \partial \Gamma)$. Remarquons que ce prolongement reste $\Gamma$-invariant et de classe $C^{\infty}$ pour la variable $x$, les dérivées successives étant continues pour $(x, \theta) \in \widetilde{X} \times(\Gamma \cup \partial \Gamma)$. Cela permet de définir, pour tout $s>0$, une famille $m^{s}$ de mesures sur $\Gamma$ en posant

$$
m_{x}^{s}(\{\gamma\})=f(x, \gamma) \nu_{x}^{s}(\{\gamma\}) .
$$

On vérifie facilement que $m_{x}^{s}$ converge faiblement vers $\mu_{x}$ quand $s$ tend vers $0^{+}$. Nous allons montrer que $\mathcal{R}(\mu)$ est la limite des nombres $\mathcal{R}\left(m^{s}\right)$. Pour cela, on écrit à nouveau les quotients $\mathcal{R}\left(m^{s}\right)$ et $\mathcal{R}(\mu)$ en changeant les notations :

$$
\begin{gathered}
\mathcal{R}\left(m^{s}\right)=\int_{X_{0}} \int_{\Gamma}\left|d r_{\theta}^{s}(x)\right|^{2} d \mu_{o}^{\prime s}(\theta) d x / \int_{X_{0}} m_{x}^{s}(\Gamma) d x, \\
\mathcal{R}(\mu)=\int_{X_{0}} \int_{\partial \Gamma}\left|d r_{\theta}(x)\right|^{2} d \mu_{o}(\theta) d x / \int_{X_{0}} \mu_{x}(\Gamma) d x,
\end{gathered}
$$

où $r_{\theta}^{s}$ et $r_{\theta}$ sont les fonctions définies par

$$
\begin{gathered}
r_{\theta}^{s}(x)^{2}=\frac{d m_{x}^{s}}{d \mu_{o}^{\prime s}}(\theta)=f(x, \theta) \sum_{\gamma} \rho\left(\gamma^{-1} x\right) e^{-C_{\theta}(\gamma(o), o)-s B_{\theta}(\gamma(o), o)}, \\
r_{\theta}(x)^{2}=\frac{d \mu_{x}}{d \mu_{o}}(\theta)=f(x, \theta) \sum_{\gamma} \rho\left(\gamma^{-1} x\right) e^{-C_{\theta}(\gamma(o), o)} .
\end{gathered}
$$

Notons que, dans les expressions ci-dessus, $\theta$ appartient à $\Gamma \cup \partial \Gamma$. Remarquons aussi que, dans les sommes ci-dessus, il n'y a qu'un nombre fini de termes non nuls. Donc $\left|d r_{\theta}^{s}(x)\right|^{2}$ converge vers $\left|d r_{\theta}(x)\right|^{2}$ uniformément pour $(x, \theta) \in X_{0} \times(\Gamma \cup \partial \Gamma)$. Rappelons aussi que $\mu_{o}^{s}$ converge faiblement vers $\mu_{o}$ et que $m_{x}^{s}$ converge faiblement vers $\mu_{x}$. De tout cela on peut déduire que $\lim _{s \rightarrow 0^{+}} \mathcal{R}\left(m^{s}\right)=\mathcal{R}(\mu)$. Or $\mathcal{R}\left(m^{s}\right) \geqslant \lambda_{1}$ d'après (2). Donc $\mathcal{R}(\mu) \geqslant \lambda_{1}$.

2ème partie : On se donne $\nu$ une famille équivariante de mesures sur $\partial \Gamma$ quelconque. On veut montrer que, pour tout $\epsilon>0$, il existe une famille équivariante de mesures $\mu$ équivalente à $\nu$

4e SÉRIE - TOME $40-2007-\mathrm{N}^{\circ} 2$ 
telle $\mathcal{R}(\mu) \leqslant \lambda_{1}+\epsilon$. On sait qu'il existe une fonction $f$ sur $\widetilde{X}, C^{\infty}$ à support compact telle que $R(f) \leqslant \lambda_{1}+\epsilon$. On peut supposer que $f$ est non nulle sur un domaine fondamental de $\Gamma$. On peut alors définir $\mu$ par

$$
\mu_{x}=\sum_{\gamma} f^{2}\left(\gamma^{-1}(x)\right) \gamma_{*} \nu_{o}
$$

où $o$ est un point quelconque. Le quotient de Rayleigh de $\mu$ peut s'écrire

$$
\mathcal{R}(\mu)=\int_{X_{0}} \int_{\partial \Gamma}\left|d s_{\theta}(x)\right|^{2} d \nu_{o}(\theta) d x / \int_{X_{0}} \mu_{x}(\partial \Gamma) d x,
$$

où $s_{\theta}$ désigne ici la fonction définie par

$$
s_{\theta}(x)^{2}=\frac{d \mu_{x}}{d \nu_{o}}(\theta)=\sum_{\gamma} f^{2}\left(\gamma^{-1}(x)\right) \frac{d\left(\gamma_{*} \nu_{o}\right)}{d \nu_{o}}(\theta) .
$$

On vérifie par un calcul simple (utilisant l'inégalité de Cauchy-Schwartz) que $\mathcal{R}(\mu) \leqslant R(f) \leqslant$ $\lambda_{1}+\epsilon$.

Remarque. - On trouve dans [10] un énoncé équivalent au théorème 3.

On peut se poser la question de l'existence et de l'unicité d'une famille équivariante de mesures $\mu$ pour laquelle la borne inférieure est atteinte dans le théorème 3, c'est-à-dire telle qu'on ait

$$
\lambda_{1}=\mathcal{R}(\mu) .
$$

Dans la section 3, on va voir que c'est le cas si $g$ est une métrique hyperbolique. La question reste ouverte pour $g$ quelconque.

Dans la fin de cette section, on étudie le cas où la borne inférieure dans le théorème 3 est atteinte par certaines familles classiques de mesures, qui sont la famille des mesures de Patterson et la famille des mesures harmoniques (ceci n'a pas de rapport avec le théorème 1).

Soit $\mu$, une quelconque famille équivariante de mesures sur $\partial \Gamma$, normalisée de telle sorte que $\int_{X_{0}} \mu_{x}(\partial \Gamma) d x=1$. Soit $r_{\theta}(x)$ la fonction définie par

$$
r_{\theta}(x)^{2}=\frac{d \mu_{x}}{d \mu_{o}}(\theta)
$$

LEMME 4.- On peut écrire

$$
\mathcal{R}(\mu)=\int_{X_{0}} \int_{\partial \Gamma} r_{\theta}(x) \Delta r_{\theta}(x) d \mu_{o}(\theta) d x,
$$

où $\Delta$ désigne le laplacien pour la variable $x$.

Démonstration. - Il s'agit d'appliquer la formule de Stokes après s'être ramené à des intégrales de fonctions à supports compacts. Pour cela, on se donne $\left(U_{i}\right)$ un recouvrement de $X$ par des ouverts difféomorphes à des boules, et $\left(\sigma_{i}\right)$ une partition de l'unité adaptée à ce recouvrement. Soit $\pi: \widetilde{X} \rightarrow X$ la projection canonique. On associe à chacun des $U_{i}$ un ouvert $\widetilde{U}_{i} \subset \widetilde{X}$ qui lui est difféomorphe via l'application $\pi$. 


$$
\begin{aligned}
\mathcal{R}(\mu) & =\int_{X_{0}} \int_{\partial \Gamma}\left|d r_{\theta}(x)\right|^{2} d \mu_{o}(\theta) d x \\
& =\sum_{i} \int_{X_{0}} \int_{\partial \Gamma}\left(d r_{\theta}(x), d\left(r_{\theta}(x) \sigma_{i}(\pi(x))\right)\right) d \mu_{o}(\theta) d x \\
& =\sum_{i} \int_{\widetilde{U}_{i}} \int_{\partial \Gamma}\left(d r_{\theta}(x), d\left(r_{\theta}(x) \sigma_{i}(\pi(x))\right)\right) d \mu_{o}(\theta) d x \\
& =\sum_{i} \int_{\widetilde{U}_{i}} \int_{\partial \Gamma} \Delta r_{\theta}(x) r_{\theta}(x) \sigma_{i}(\pi(x)) d \mu_{o}(\theta) d x \\
& =\int_{X_{0}} \int_{\partial \Gamma} r_{\theta}(x) \Delta r_{\theta}(x) d \mu_{o}(\theta) d x .
\end{aligned}
$$

LEMME 5. - Si $\mathcal{R}(\mu)=\lambda_{1}$, alors on a pour tout $\theta \in \partial \Gamma$

$$
\Delta r_{\theta}=\lambda_{1} r_{\theta}
$$

Démonstration. - Soit $f: \widetilde{X} \times \partial \Gamma \rightarrow \mathbb{R}$, une fonction $C^{\infty}$ en la variable $x$, telle que

$$
\int_{X_{0}} \int_{\partial \Gamma} f(x, \theta) d \mu_{x}(\theta) d x=0 .
$$

Pour $t$ suffisamment proche de 0 , on définit la famille équivariante de mesures $\mu^{t}$ par

$$
\frac{d \mu_{x}^{t}}{d \mu_{x}}(\theta)=1+t f(x, \theta)
$$

La condition (4) signifie que $\mu^{t}$ est normalisé. Comme $\mathcal{R}(\mu)$ est minimale, on en déduit que

$$
\left.\frac{d}{d t}\right|_{t=0} \mathcal{R}\left(\mu^{t}\right)=0
$$

La quantité $\mathcal{R}\left(\mu^{t}\right)$ s'écrit (en n'écrivant pas les variables pour alléger)

$$
\mathcal{R}\left(\mu^{t}\right)=\int_{X_{0}} \int_{\partial \Gamma}|d(r \sqrt{1+t f})|^{2} d \mu_{o}(\theta) d x .
$$

On en déduit que

$$
\begin{aligned}
0 & =\int_{X_{0}} \int_{\partial \Gamma}(d(r f), d r) d \mu_{0}(\theta) d x \\
& =\int_{X_{0}} \int_{\partial \Gamma} f r \Delta r d \mu_{0}(\theta) d x .
\end{aligned}
$$

Comme $f$ est une fonction quelconque vérifiant (4), on a alors $\Delta r$ est égal à $r$ multipliée par une constante. D'après (3), la constante est $\lambda_{1}$.

$4^{\text {e }}$ SÉRIE - TOME $40-2007-\mathrm{N}^{\circ} 2$ 
La famille des mesures de Patterson. - Rappelons que la famille des mesures de Patterson, notée $p$, est l'unique famille équivariante de mesures sur $\partial \Gamma$ telle qu'il existe une constante $h$ telle qu'on ait

$$
\frac{d p_{x}}{d p_{o}}(\theta)=e^{-h B(x, \theta)},
$$

où $B(x, \theta)$ désigne la fonction de Busemann normalisée par $B(o, \theta)=0$ (voir par exemple [18]). Cette constante $h$ est l'entropie volumique de $X$ pour la métrique $g$. Un calcul simple montre que $\mathcal{R}(p)=h^{2} / 4$, et donc que $\lambda_{1} \leqslant h^{2} / 4$. Le théorème suivant illustre le lemme 5 . Il a été établi auparavant à la suite des travaux de Ledrappier, Foulon, Labourie, Besson, Courtois, Gallot [16, 7,2] :

THÉORÈME 6. - Si $\lambda_{1}=h^{2} / 4$, alors la métrique est localement symétrique.

Démonstration. - Si $\lambda_{1}=h^{2} / 4$, on a donc $\mathcal{R}(p)=h^{2} / 4=\lambda_{1}$, et alors, d'après le lemme 5 , on a

$$
\Delta e^{-\frac{h}{2} B}=\frac{h^{2}}{4} e^{-\frac{h}{2} B} .
$$

De cela on peut déduire facilement que $\Delta B=h$. Or on sait grâce à [7] et [2] que cela équivaut à dire que la métrique est localement symétrique.

La famille des mesures harmoniques. - La famille des mesures harmoniques $\nu=\left(\nu_{x}\right)$ est l'unique famille équivariante de mesures sur $\partial \Gamma$ telle que, pour tout $\theta \in \partial \Gamma$, la fonction $x \rightarrow \frac{d \nu_{x}}{d \nu_{o}}(\theta)$ soit harmonique (voir par exemple [1]). Le nombre

$$
\beta=4 \mathcal{R}(\nu)
$$

est l'entropie stochastique de $X$ (voir [16,11]). On a naturellement l'inégalité

$$
\beta \geqslant 4 \lambda_{1} .
$$

Le théorème suivant est encore une conséquence des travaux Ledrappier, Foulon, Labourie, Besson, Courtois, Gallot (voir [14,7,2], ou aussi [10])

THÉORÈME 7. - Soit $X$ une variété riemannienne compacte à courbure strictement négative. Si $\beta=4 \lambda_{1}$, alors la métrique est localement symétrique.

Démonstration. - Soit $r_{\theta}(x)$ la fonction telle que

$$
r_{\theta}(x)^{2}=\frac{d \nu_{x}}{d \nu_{o}}(\theta)
$$

Si on a $\mathcal{R}(\nu)=\lambda_{1}$, alors d'après le lemme $5, \Delta r=\lambda_{1} r$. De cela et du fait que $\Delta\left(r^{2}\right)=0$, on déduit facilement que

$$
|d(\ln r)|=\sqrt{\lambda_{1}}
$$

Donc, pour tout $\theta \in \partial \Gamma$, la différentielle de la fonction $x \rightarrow \ln (r(x, \theta))$, définie sur $\widetilde{X}$, est de norme constante. Or seules les fonctions de Busemann ont la propriété d'avoir leur différentielle de norme constante égale à 1 sur $\widetilde{X}$ tout entier. Donc on peut écrire

$$
\ln (r(x, \theta))=\sqrt{\lambda_{1}} B\left(x, \theta^{\prime}\right) .
$$


où $\theta^{\prime}$ est un point de $\partial \Gamma$ dépendant de $\theta$. En fait, on a tout simplement que $\theta^{\prime}=\theta$ car la fonction $\theta \rightarrow \theta^{\prime}$ qui est continue et $\Gamma$-équivariante ne peut être que l'identité. Finalement, on a montré que

$$
\frac{d \nu_{x}}{d \nu_{o}}(\theta)=\exp \left(-2 \sqrt{\lambda_{1}} B(x, \theta)\right)
$$

ce qui implique que $\nu$ est la famille des mesures de Patterson, et que $2 \sqrt{\lambda_{1}}=h$. Le théorème 6 permet de conclure.

\section{Preuve du théorème 1}

L'avantage de la description du $\lambda_{1}(g)$ avec les familles équivariantes de mesures sur $\partial \Gamma$ est qu'on peut espérer trouver une famille $\mu_{0}$ telle que $\mathcal{R}\left(\mu_{0}\right)$ soit minimal, alors qu'on sait bien qu'il n'existe pas de fonction sur $\widetilde{X}$ dont le quotient de Rayleigh est minimal.

Dans cette section, $X$ est une variété compacte de dimension $n$ qui admet une métrique hyperbolique $g_{0}$. On va ici comparer $\lambda_{1}\left(g_{0}\right)$ et $\lambda_{1}(g)$, lorsque $g$ est une autre métrique de même volume que $g_{0}$.

On fixe une origine $o \in \widetilde{X}$. On note $B(x, \theta)$ la fonction de Busemann pour $g_{0}$, normalisée par $B(o, \theta)=0$. L'entropie volumique de $X$ pour la métrique $g_{0}$ est égale à $n-1$. On note $P=\left(P_{x}\right)$ la famille des mesures de Patterson pour $g_{0}$, caractérisée par

$$
\frac{d P_{x}}{d P_{o}}(\theta)=e^{-(n-1) B(x, \theta)} .
$$

Nous la normalisons de telle sorte que

$$
\int_{X_{0}} P_{x}(\partial \Gamma) d x=1 .
$$

On vérifie que

$$
\mathcal{R}_{g_{0}}(P)=\frac{(n-1)^{2}}{4} .
$$

Rappelons que $\lambda_{1}\left(g_{0}\right)=(n-1)^{2} / 4$, ce qui peut se déduire par exemple de la proposition 9 .

Toute famille $\mu$ équivalente à $P$ est caractérisée par les relations de densité

$$
\frac{d \mu_{x}}{d P_{x}}(\theta)=e^{-f(x, \theta)}
$$

où $f$ est une fonction équivariante et $C^{\infty}$ en la variable $x$ au sens où ses jets sont continus pour $(x, \theta)$. Enfin, dans ce qui suit, le symbole $d x$ fera référence à la mesure induite par $g_{0}$, et les notations $(\cdot, \cdot)$ et $|\cdot|^{2}$ désigneront des produits scalaires pour la métrique $g_{0}$.

Comportement de $\lambda_{1}(g)$ dans la tranche d'Ebin. - On considère $\left(X, g_{0}\right)$ une variété hyperbolique réelle.

DÉfinition 8 ([3] et [5]). - On définit la tranche d'Ebin de $g_{0}$ comme l'espace des métriques $g$ sur $X$ qui s'écrivent

$$
g=g_{0}\left(e^{H} \cdot, \cdot\right)
$$

$4^{\mathrm{e}}$ SÉRIE - TOME $40-2007-\mathrm{N}^{\circ} 2$ 
où $H$ est une section du fibré des endomorphismes symétriques du fibré $T X$ muni de la métrique $g_{0}$, qui vérifie les conditions

(i) $\operatorname{trace}(H)=0$,

(ii) $\delta H=0$.

Dans cette définition, la section $H$ peut s'identifier à un vecteur tangent à l'espace des métriques au point $g_{0}$. La condition (i) signifie alors que $H$ est orthogonale à la classe conforme de $g_{0}$, tandis que la condition (ii) signifie que $H$ est orthogonale à l'orbite de $g_{0}$ sous l'action des difféomorphismes de $X$.

Notons aussi que, pour une métrique $g$ dans la tranche d'Ebin de $g_{0}$, les mesures riemanniennes induites par $g$ et $g_{0}$ sont les mêmes ( $\operatorname{car} \operatorname{det}\left(e^{H}\right)=1$ ). Rappelons aussi que la condition $\delta H=0$ revient à dire que, pour tout champ de vecteurs $X$, on a

$$
\int_{X}(H, D X) d x=0,
$$

ou encore, en notant $h=g_{0}(H \cdot, \cdot)$, pour toute 1 -forme $\alpha$ sur $X$, on a

$$
\int_{X}(h, D \alpha) d x=0 .
$$

La proposition suivante permet d'étudier le comportement de $\lambda_{1}(g)$ dans la tranche d'Ebin.

PROposition 9. - Soit $\left(X, g_{0}\right)$ une variété hyperbolique réelle de dimension $n \geqslant 3$. Il existe un $C^{0}$ voisinage $\mathcal{V}$ de $g_{0}$ dans la tranche d'Ebin tel que, pour toute métrique $g \in \mathcal{V}$ et pour toute famille équivariante de mesures $\mu$ équivalente à $P$, on ait

$$
\mathcal{R}_{g}(\mu) \geqslant \mathcal{R}_{g_{0}}(P) .
$$

Par conséquent, on a

$$
\lambda_{1}(g) \geqslant \lambda_{1}\left(g_{0}\right)=\frac{(n-1)^{2}}{4} .
$$

De plus, l'égalité $\lambda_{1}(g)=\lambda_{1}\left(g_{0}\right)$ est atteinte si et seulement si $g=g_{0}$.

Démonstration. - Soit $g=g_{0}\left(e^{H} \cdot, \cdot\right)$ dans la tranche d'Ebin, et soit $\mu$ une famille équivalente à $P$. On peut supposer, quitte à renormaliser, que

$$
\int_{X_{0}} \mu_{x}(\partial \Gamma) d x=1 .
$$

On peut écrire

$$
\frac{d \mu_{x}}{d P_{x}}(\theta)=e^{-f(x, \theta)}
$$

Donc

$$
\frac{d \mu_{x}}{d P_{o}}(\theta)=e^{-f(x, \theta)-(n-1) B(x, \theta)} .
$$


Pour exprimer $\mathcal{R}_{g}(\mu)$, on a besoin d'écrire le produit scalaire pour $g$ à l'aide de celui pour $g_{0}$, noté $(\cdot, \cdot)$, et de $e^{H}$ : le produit scalaire ponctuel pour $g$ de deux 1-formes $a$ et $b$ s'écrit :

$$
\left(a e^{-H}, b\right), \quad \text { ou aussi }\left(a, b e^{-H}\right) .
$$

On a donc (en n'écrivant plus les variables $x$ et $\theta$ pour alléger) :

$$
\mathcal{R}_{g}(\mu)=\frac{1}{4} \int_{X_{0}} \int_{\partial \Gamma}\left((d f+(n-1) d B),(d f+(n-1) d B) e^{-H}\right) d \mu_{x}(\theta) d x .
$$

Donc

$$
\begin{aligned}
\mathcal{R}_{g}(\mu)-\mathcal{R}_{g_{0}}(P)= & \frac{1}{4} \int_{X_{0}} \int_{\partial \Gamma}\left[\left(d f, d f e^{-H}\right)+2(n-1)\left(d B, d f e^{-H}\right)\right. \\
& \left.+(n-1)^{2}\left(d B, d B\left[e^{-H}-\mathrm{id}\right]\right)\right] d \mu_{x}(\theta) d x .
\end{aligned}
$$

On veut montrer que cette quantité est positive. Dans cette expression, le terme entre crochets s'écrit sous la forme

$$
(a, a A)+(b, a B)+(b, b C),
$$

où $a$ et $b$ désignent les 1 -formes $d f$ et $d B$ et $A, B, C$ désignent respectivement les endomorphismes symétriques $e^{-H}, 2(n-1) e^{-H}$ et $(n-1)^{2}\left[e^{-H}-\mathrm{id}\right]$. Ce terme entre crochets n'a pas de raison d'être une fonction positive car, par exemple, l'endomorphisme $C=(n-1)^{2}\left[e^{-H}-\mathrm{id}\right]$ n'est pas toujours défini positif. On va transformer l'expression (5) de manière à ce que le terme entre crochets soit une fonction positive (lorsque $H$ est $C^{0}$-proche de 0 ).

On introduit pour cela la 1 -forme $\alpha$ suivante sur $\tilde{X}$ : pour tout vecteur $u$ tangent en $x$, on pose

$$
\alpha(x)(u)=\int_{\partial \Gamma} d B(x, \theta)(u) d \mu_{x}(\theta) .
$$

On réécrit cela ainsi :

$$
\alpha(x)=\int_{\partial \Gamma} d B(x, \theta) e^{-(f+(n-1) B)(x, \theta)} d P_{o}(\theta) .
$$

Cette 1 -forme passe au quotient et définit une 1 -forme sur $X$. On calcule sa dérivée covariante (pour la métrique $g_{0}$ ) : c'est le 2-tenseur

$$
D \alpha(x)=\int_{\partial \Gamma}[D d B-(d f+(n-1) d B) \otimes d B] d \mu_{x}(\theta) .
$$

Comme $\left(\widetilde{X}, g_{0}\right)$ est l'espace hyperbolique de dimension $n$, on a la relation

$$
D d B=g_{0}-d B \otimes d B .
$$

Donc

$$
D \alpha(x)=\int_{\partial \Gamma}\left[g_{0}-d f \otimes d B-n d B \otimes d B\right] d \mu_{x}(\theta) .
$$

$4^{\text {e }}$ SÉRIE - TOME 40 - $2007-\mathrm{N}^{\circ} 2$ 
On sait que la divergence d'une 1-forme sur $X$ est d'intégrale nulle. Ceci appliqué à $\alpha$ donne la relation

$$
\int_{X_{0}} \int_{\partial \Gamma}(d f, d B) d \mu_{x}(\theta) d x=-\int_{X} \operatorname{trace} D \alpha=0 .
$$

D'autre part, on contracte $D \alpha$ avec le 2-tenseur $h=g_{0}(H \cdot, \cdot)$ et on intègre sur $X$. Comme $\delta H=0$ et comme $\left(h, g_{0}\right)=\operatorname{trace} H=0$, on obtient la relation

$$
0=-\int_{X}(h, D \alpha)=\int_{X_{0}} \int_{\partial \Gamma}[(d B, d f H)+n(d B, d B H)] d \mu_{x}(\theta) d x .
$$

Alors en combinant l'expression (5) avec les relations (6) et (7) on obtient l'expression

$$
\begin{aligned}
& \mathcal{R}_{g}(\mu)-\mathcal{R}_{g_{0}}(P) \\
& =\frac{1}{4} \int_{X_{0}} \int_{\partial \Gamma}\left[\left(d f, d f e^{-H}\right)+2(n-1)\left(d B, d f\left[e^{-H}-\mathrm{id}+\frac{(n-1)}{2 n} H\right]\right)\right. \\
& \left.\quad+(n-1)^{2}\left(d B, d B\left[e^{-H}-\mathrm{id}+H\right]\right)\right] d \mu_{x}(\theta) d x .
\end{aligned}
$$

Cette fois le terme entre crochets est une fonction positive lorsque $H$ est $C^{0}$-proche de 0 . En effet, le terme entre crochets s'écrit sous la forme

$$
(a, a A)+(b, a B)+(b, b C)
$$

et il est positif car le «discriminant» $\left(B A^{-1} B-4 C\right)$ est un endomorphisme symétrique négatif. En effet, soit $x \in \widetilde{X}$ et soit $\left(e_{i}\right)$ une base de $T_{x} \widetilde{X}$ qui diagonalise $H$ et soient $\left(\lambda_{i}\right)$ les valeurs propres associées. Alors,

- $\left(e_{i}\right)$ diagonalise $A$ et les valeurs propres sont $e^{-\lambda_{i}}=1+\mathrm{o}(1)$,

- $\left(e_{i}\right)$ diagonalise $B$ et les valeurs propres sont $2(n-1)\left[e^{-\lambda_{i}}-1+\frac{n-1}{2 n} \lambda_{i}\right]=-\frac{(n-1)(n+1)}{n} \lambda_{i}$ $+\mathrm{o}\left(\left|\lambda_{i}\right|\right)$

- $\left(e_{i}\right)$ diagonalise $C$ et les valeurs propres sont $(n-1)^{2}\left[e^{-\lambda_{i}}-1+\lambda_{i}\right]=\frac{(n-1)^{2}}{2} \lambda_{i}^{2}+\mathrm{o}\left(\left|\lambda_{i}\right|^{2}\right)$, donc $\left(e_{i}\right)$ diagonalise le «discriminant» $B A^{-1} B-4 C$ et les valeurs propres ont pour développement limité

$$
(n-1)^{2}\left[\frac{(n+1)^{2}}{n^{2}}-2\right] \lambda_{i}^{2}+\mathrm{o}\left(\left|\lambda_{i}\right|^{2}\right) .
$$

Elles sont bien négatives lorsque $H$ est $C^{0}$-proche de 0 et $n \geqslant 3$.

Cas d'égalité : Supposons maintenant que

$$
\lambda_{1}(g)=\frac{(n-1)^{2}}{4}
$$

On doit montrer que, si $H$ est $C^{0}$-proche de 0 , alors $H=0$.

Par hypothèse, il existe une suite $\mu^{k}$ de familles équivariantes de mesures équivalentes à $P$, normalisées, telles que

$$
\lim _{k \rightarrow \infty} \mathcal{R}_{g}\left(\mu^{k}\right)-\mathcal{R}_{g_{0}}(P)=0
$$


Soit $f_{k}$ tel que

$$
\frac{d \mu_{x}^{k}}{d P_{x}}(\theta)=e^{-f_{k}(x, \theta)}
$$

Écrivons à nouveau l'équation (8)

$$
\begin{aligned}
\mathcal{R}_{g}\left(\mu^{k}\right)-\mathcal{R}_{g_{0}}(P)= & \frac{1}{4} \int_{X_{0}} \int_{\partial \Gamma}\left[\left(d f_{k}, d f_{k} e^{-H}\right)+2(n-1)\left(d B, d f_{k}\left[e^{-H}-\mathrm{id}+\frac{(n-1)}{2 n} H\right]\right)\right. \\
& \left.+(n-1)^{2}\left(d B, d B\left[e^{-H}-\mathrm{id}+H\right]\right)\right] d \mu_{x}^{k}(\theta) d x
\end{aligned}
$$

Le terme entre crochets s'écrit encore sous la forme

$$
(a, a A)+(b, a B)+(b, b C)
$$

et on a déjà montré qu'il est positif lorsque $H$ est $C^{0}$-proche de 0 . On peut montrer plus : soit $\eta>0$, alors le terme

$$
(1-\eta)(a, a A)+(b, a B)+(1-\eta)(b, b C)
$$

est positif lorsque $H$ est $C^{0}$ proche de 0 et $\eta$ suffisamment petit (et $n \geqslant 3$ ) car alors le «discriminant» $(1-\eta)^{-1} B A^{-1} B-4(1-\eta) C$ est symétrique négatif. On peut donc écrire

$$
\begin{aligned}
(a, a A)+(b, a B)+(b, b C)= & \eta(a, a A)+\eta(b, b C)+(1-\eta)(a, a A) \\
& +(b, a B)+(1-\eta)(b, b C) .
\end{aligned}
$$

De plus, comme $A=e^{-H}$ et $C=e^{-H}-\mathrm{id}+H$, on en déduit que les endomorphismes $A-\frac{1}{2}$ id et $C-\frac{1}{4} H^{2}$ sont symétriques positifs lorsque $H$ est $C^{0}$-proche de 0 . On peut donc écrire

$$
\begin{aligned}
(a, a A)+(b, a B)+(b, b C)= & \left(a, a\left(A-\frac{1}{2} \mathrm{id}\right)\right)+\eta\left(b, b\left(C-\frac{1}{4} H^{2}\right)\right) \\
& +(1-\eta)(a, a A)+(b, a B)+(1-\eta)(b, b C) \\
& +\frac{\eta}{2}|a|^{2} \\
& +\frac{\eta}{4}|b H|^{2},
\end{aligned}
$$

et dans le terme de droite, chaque ligne est positive. De tout ceci, on déduit que

$$
\lim _{k} \int_{X_{0}} \int_{\partial \Gamma}\left|d f_{k}(x, \theta)\right|^{2} d \mu_{x}^{k}(\theta) d x=0,
$$

et

$$
\lim _{k} \int_{X_{0}} \int_{\partial \Gamma}|d B(x, \theta) H|^{2} d \mu_{x}^{k}(\theta) d x=0 .
$$

Considérons alors la suite des mesures $d \mu_{x}^{k}(\theta) d x$ sur le compact $\tilde{X} \times \partial \Gamma$. Ces mesures sont invariantes pour l'action diagonale de $\Gamma$ et induisent des mesures finies sur le quotient qui est compact. De plus, ces mesures sont de masse 1, et on peut donc extraire une sous-suite qui 
converge faiblement vers une certaine mesure finie que l'on va noter $m$ pour l'instant. On peut voir $m$ comme une mesure $\Gamma$-invariante sur $\widetilde{X} \times \partial \Gamma$. On a donc

$$
\int_{X_{0}} \int_{\partial \Gamma}|d B(x, \theta) H|^{2} d m(x, \theta)=0
$$

Si $m$ est une mesure qui charge les ouverts, alors cela veut dire que $d B(x, \theta) H=0$ pour tout $(x, \theta)$, et donc $H=0$. Donc, pour conclure la preuve, il suffit de montrer que $m$ charge les ouverts. Nous allons en fait montrer que $m$ n'est autre que la mesure $d P_{x}(\theta) d x$ (qui charge les ouverts, bien sûr).

Soit $c$ une fonction sur $\widetilde{X} \times \partial \Gamma$ de classe $C^{2}$, i.e. telle que ses dérivées premières et secondes pour la variable $x$ sont des fonctions continues de $(x, \theta)$. On note $\Delta c$ le laplacien de $c$ pour la variable $x$ induit par la métrique $g_{0}$. La mesure $d P_{x}(\theta) d x$ est une mesure harmonique pour le feuilletage stable, ce qui signifie qu'on a

$$
\int_{\widetilde{X} \times \partial \Gamma} \Delta c(x, \theta) d P_{x}(\theta) d x=0
$$

pour toute fonction $c$ à support compact. Cela se vérifie facilement en écrivant $d P_{x}(\theta) d x=$ $\exp (-(n-1) B(x, \theta)) d P_{o}(\theta) d x$. Dans [15] ou dans [8], on montre qu'il n'y a qu'une seule mesure harmonique pour le feuilletage stable. Il nous suffit donc de montrer que $m$ est harmonique pour le feuilletage stable : pour toute fonction $c$, de classe $C^{2}$, à support compact, on peut écrire (en omettant les variables $x$ et $\theta$ pour alléger)

$$
\int_{\widetilde{X} \times \partial \Gamma} \Delta c d m=\lim _{k} \int_{\widetilde{X}} \int_{\partial \Gamma} \Delta c \exp \left(-f_{k}-(n-1) B\right) d P_{o}(\theta) d x .
$$

Or,

$$
\begin{aligned}
\int_{\widetilde{X}} & \int_{\partial \Gamma} \Delta c \exp \left(-f_{k}-(n-1) B\right) d P_{o}(\theta) d x \\
= & \int_{\widetilde{X}} \int_{\partial \Gamma}\left(d c, d e^{-f_{k}-(n-1) B}\right) d P_{o}(\theta) d x \\
= & \int_{\widetilde{X}} \int_{\partial \Gamma}\left[\left(e^{-f_{k}} d c, d e^{-(n-1) B}\right)+\left(d c,-d f_{k}\right) e^{-f_{k}-(n-1) B}\right] d P_{o}(\theta) d x \\
= & \iint_{0}\left(d\left(c e^{-f_{k}}\right), d e^{-(n-1) B}\right) d P_{o}(\theta) d x+\iint\left(d f_{k}, c(n-1) d B-d c\right) d \mu_{x}^{k}(\theta) d x
\end{aligned}
$$

Dans la dernière ligne, le premier terme est nul car $\Delta e^{-(n-1) B}=0$, et le deuxième tend vers zéro quand $k$ tend vers l'infini, car comme $c$ et $d c$ sont bornées et à support compact, on peut utiliser l'inégalité de Cauchy-Schwartz et majorer ce terme par une constante fois

$$
\left(\int_{X_{0}} \int_{\partial \Gamma}\left|d f_{k}\right|^{2} d \mu_{x}^{k}(\theta) d x\right)^{1 / 2}
$$


qui tend vers zéro, comme on l'a vu avant. Donc

$$
\int_{\widetilde{X} \times \partial \Gamma} \Delta c d m=0
$$

donc $m$ est harmonique pour le feuilletage stable, ce qui termine la preuve.

Remarque. - La proposition 9 est inspirée de la proposition 4.1 dans [3], qui affirme que $g_{0}$ est un minimum local strict dans sa tranche d'Ebin pour l'invariant $T_{2}(g)$ qui peut être défini ici par

$$
T_{2}(g)=\inf \left\{R(\mu), \mu \text { équivalente à } P \text {, et } \mu_{x}(\partial \Gamma)=1 \text { pour tout } x\right\} .
$$

Mais on a $\lambda_{1}(g) \leqslant T_{2}(g)$, et donc la proposition 4.1 de [3] seule ne suffit pas à traiter le cas de $\lambda_{1}(g)$.

Comportement de $\lambda_{1}(g)$ dans la classe conforme (cf. [4]). - Maintenant on va étudier le comportement de $\lambda_{1}(g)$ dans la classe conforme de $g_{0}$ : Soit $g$ une métrique conforme à $g_{0}$, différente de $g_{0}$ mais de même volume que $g_{0}$. Elle s'écrit

$$
g=k^{2} g_{0},
$$

où $k$ est une fonction telle que $\int_{X} k^{n}=\operatorname{Vol}\left(g_{0}\right)$. Pour toute 1-forme $\alpha$ sur $X$, le carré scalaire de $\alpha$ pour $g$ s'écrit :

$$
k^{-2}|\alpha|^{2}
$$

Dans la proposition suivante, on compare les quotients de Rayleigh de $P$ selon les métriques $g$ et $g_{0}$ :

PROPOSITION 10. - Soit $g$ une métrique conforme à $g_{0}$ et de même volume, mais différente de $g_{0}$. Alors on a

$$
\mathcal{R}_{g}(P)<\mathcal{R}_{g_{0}}(P) .
$$

Démonstration. - Comme $g_{0}$ est une métrique hyperbolique, l'application $x \rightarrow P_{x}(\partial \Gamma)$ est constante. Par conséquent, on a

$$
\mathcal{R}_{g}(P)=\frac{(n-1)^{2}}{4} \int_{X} k(x)^{n-2} d x / \int_{X} d x ;
$$

donc, en utilisant l'inégalité de Hölder,

$$
\mathcal{R}_{g}(P)<\frac{(n-1)^{2}}{4}=\mathcal{R}_{g_{0}}(P) .
$$

COROLLAIRE 11. - $\lambda_{1}(g)$ est un maximum strict au point $g_{0}$ dans la classe conforme de $g_{0} \grave{a}$ volume constant.

\section{RÉFÉRENCES}

[1] Anderson M., Schoen R., Positive harmonic functions on complete manifolds of negative curvature, Ann. of Math. 121 (1985) 429-461.

$4^{\text {e }}$ SÉRIE - TOME $40-2007-\mathrm{N}^{\circ} 2$ 
[2] Besson G., Courtois G., Gallot S., Entropies et rigidités des espaces localement symétriques de courbure strictement négative, Geom. Funct. Anal. 5 (5) (1995) 731-799.

[3] Besson G., Courtois G., Gallot S., Les variétés hyperboliques sont des minima locaux de l'entropie topologique, Invent. Math 117 (3) (1994) 403-445.

[4] Besson G., Courtois G., Gallot S., Volume et entropie minimale des espaces localement symétriques, Invent. Math. 103 (2) (1991) 417-445.

[5] BESSE A.L., Einstein manifolds, Ergeb. Math. Grenzgeb., vol. 10, Springer-Verlag, Berlin/ Heidelberg/New York, 1987.

[6] Bourdon M., Structure conforme au bord et flot géodésique d'un CAT (-1)-espace, L'enseignement mathématique 41 (1995) (1985) 63-102.

[7] Foulon P., LABOURIE F., Sur les variétés compactes asymptotiquement harmoniques, Invent. Math. 109 (1992) 97-111.

[8] GaRnett L., Foliations, the ergodic theorem and Brownian motion, J. Funct. Anal. 51 (1983) 285311.

[9] Ghys É., DE La Harpe P., Sur les groupes hyperboliques d'après Mikhael Gromov, Progress in Mathematics, vol. 83, Birkhäuser Boston, Inc., Boston, MA, 1990.

[10] HamenstäDt U., Harmonic measures, Hausdorff measures and positive eigenfunctions, J. Differential Geom. 44 (1996) 1-31.

[11] KAIMANOVICH V.A., Brownian motion and harmonic functions on covering manifolds. An entropy approach, English translation Soviet Math. Dokl. 33 (1986) (1985) 812-816.

[12] LEDRAPPIER F., Structure au bord des variétés à courbure négative, in: Séminaire de Théorie Spectrale et Géométrie, No. 13, année 1994-1995, Univ. Grenoble I, Saint-Martin-d'Hères, 1995, pp. 97-122.

[13] LEDRAPPIER F., Harmonic 1-forms on the stable foliation, Bol. Soc. Brasil. Mat. (N.S.) 25 (2) (1994) $121-138$.

[14] LeDrAPPIER F., A heat kernel characterization of asymptotic harmonicity, Proc. Amer. Math. Soc. 118 (1993) 1001-1004.

[15] LEDRAPPIER F., Ergodic properties of the stable foliations, in: Ergodic Theory and Related Topics III, Güstrow, 1990, in: Lecture Notes in Math., vol. 1514, Springer-Verlag, Berlin, 1992, pp. 131-145.

[16] LEDRAPPIER F., Harmonic measures and Bowen-Margulis measures, Israel J. Math. 71 (1) (1990) 275-287.

[17] LEDRAPPIER F., Ergodic properties of Brownian motion on covers of compact negatively-curve manifolds, Bol. Soc. Brasil. Mat. 19 (1) (1990) 115-140.

[18] Roblin T., Ergodicité et équidistribution en courbure négative, Memoires de la SMF 95 (2003).

\footnotetext{
Olivier MOHSEN

École Polytechnique

Centre de Mathématiques Laurent Schwartz

91128 Palaiseau cedex, France

E-mail : mohsen@math.polytechnique.fr
} 